\title{
Probing the Buried Magnetic Interfaces
}

Wenqing Liu, ${ }^{1,2,3}$ Qionghua Zhou, ${ }^{4}$ Qian Chen, ${ }^{4}$ Daxin Niu, ${ }^{2}$ Yan Zhou, ${ }^{1,3}$ Yongbing Xu, ${ }^{1,2,} *$ Rong Zhang, , * Jinlan Wang, ${ }^{4}$ * and Gerrit van der Laan ${ }^{5}$

${ }^{1}$ York-Nanjing Joint Center (YNJC) for spintronics and nanoengineering, School of Electronics Science and Engineering, Nanjing University, Nanjing 210093, China

2 Spintronics and Nanodevice Laboratory, Department of Electronics, University of York, York YO10 $5 \mathrm{DD}, \mathrm{UK}$

${ }^{3}$ Department of Physics, The University of Hong Kong, Pokfulam, Hong Kong

${ }^{4}$ Department of Physics, Southeast University, Nanjing 211189, China

${ }^{5}$ Magnetic Spectroscopy Group, Diamond Light Source, Didcot OX11 0DE, UK

* Authors to whom correspondence should be addressed. Electronic addresses: yongbing.xu@york.ac.uk, rzhang@nju.edu.cn, and jlwang@seu.edu.cn 


\section{Abrupt interfaces}

In addition to the intermixing interfaces, described in the main paper, also the abrupt configurations were calculated. Unlike the intermixing interface, in which the bcc FM is partly continuing into the SC, the abrupt interface aligns the FM atop GaAs slab but without entering it, as illustrated in Fig. S1. The formation energy, $H$, of the intermixing and the abrupt interfaces was calculated using

$$
H=E_{t o t}-E_{G a A s}-\sum_{i} N_{i} \mu_{i}
$$

where $E_{\mathrm{tot}}, E_{\mathrm{GaAs}}, N_{i}$, and $\mu_{\mathrm{i}}$ represent the total energy of the unit cell, total energy of GaAs substrate, number of FM atoms, and chemical potential of species $i$, respectively. The calculation suggests that all the superstructures favor an intermixed interface with $\Delta H=H^{\text {abrupt }}$ $H^{\text {intermixing }}=116,38,272$, and $229 \mathrm{meV} /($ unit cell) for $7 \mathrm{ML} \mathrm{Fe} / 1 \mathrm{ML} \mathrm{Co} / \mathrm{GaAs}, 8 \mathrm{ML} \mathrm{Co} / \mathrm{GaAs}$, $7 \mathrm{ML} \mathrm{Co} / 1 \mathrm{ML} \mathrm{Ni} / \mathrm{GaAs}$, and $8 \mathrm{ML} \mathrm{Ni} / \mathrm{GaAs}$, respectively. Whilst the formation of abrupt $\mathrm{Ni} / \mathrm{GaAs}$ can be neglected, it is not a decisive advantage for the $\mathrm{Co} / \mathrm{GaAs}$ interface. The $m_{\text {spin }}$ of the Co atoms, based on the abrupt configurations, were calculated and the results, presented in Fig. S2, show similar trends as the intermixing interfaces as described in the main paper. Again, the calculated $m_{\text {spin }}$ of the interfacial $\mathrm{FM}_{2}$ gives small deviations between the $\mathrm{FM}_{1} / \mathrm{FM}_{2} / \mathrm{SC}$ and $\mathrm{FM}_{2} / \mathrm{SC}$ structures, i.e., $<0.1 \mu_{\mathrm{B}} /$ atom when exchanging the topmost Co by Fe. In agreement with the experiments and the intermixing calculations, the abrupt interfaces also reveal a wellretained magnetization of the interfacial Co on GaAs(100), i.e., $1.43 \mu_{\mathrm{B}} /$ atom. This confirms the high validity of the selected method, even with the coexistence of the secondary phases, e.g., abrupt $\mathrm{Co} / \mathrm{GaAs}$ interfaces.

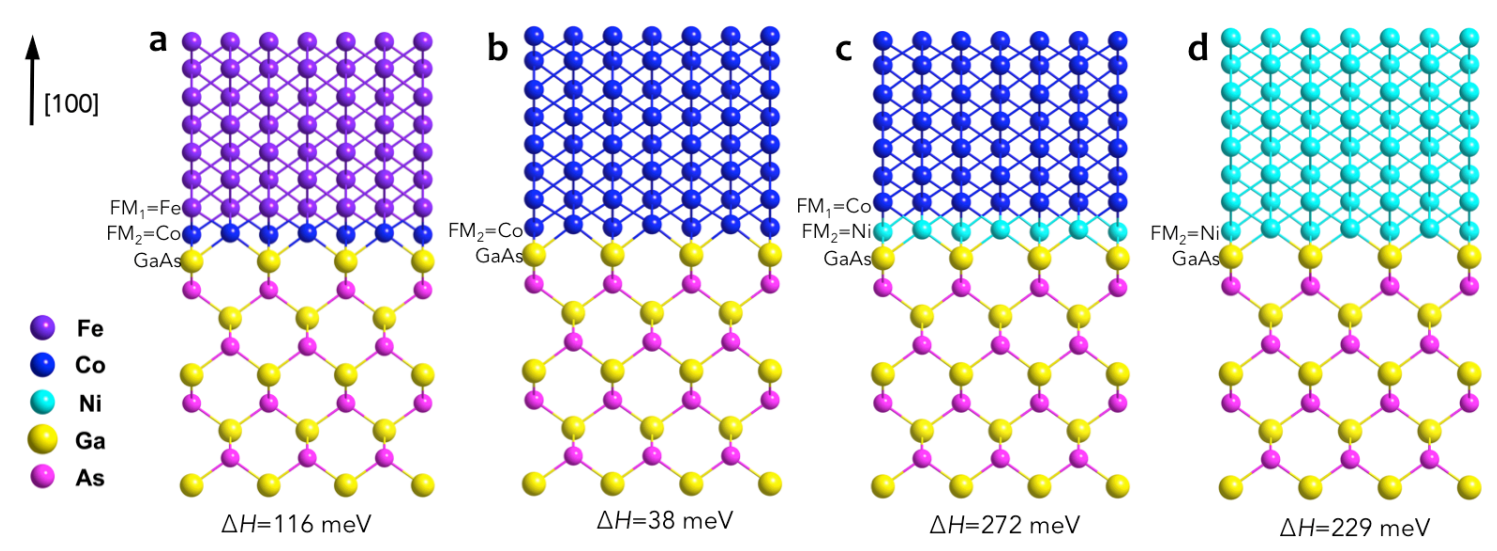

Figure S1. Side view of the FM/SC superstructures with abrupt interfaces, (a) $7 \mathrm{ML}$ Fe/1 ML Co, (b) $8 \mathrm{ML}$ $\mathrm{Co}$, (c) $7 \mathrm{ML} \mathrm{Co} / 1 \mathrm{ML} \mathrm{Ni}$, and (d) $8 \mathrm{MLNi}$ atop $\mathrm{GaAs}(100)$ and their $\Delta H$ values. 


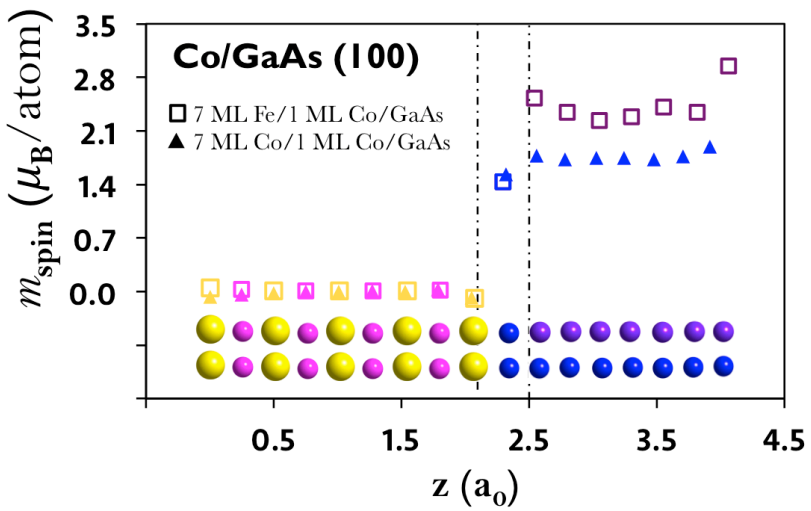

Figure S2. Calculated $m_{\text {spin }}$ for the abrupt Co/GaAs interface versus the distance $z$ along the [100] direction. The distance is normalized to the lattice constant of GaAs $\left(a_{0}=5.654 \AA\right.$ ). The colored spheres at the bottom indicate the position of each atomic layer. The dash-dotted lines indicate the interfacial or the $\mathrm{FM}_{2}$ region.

\section{Band structures}

To gain insight into the detailed orbital hybridization of the $\mathrm{Co} / \mathrm{GaAs}$ and $\mathrm{Ni} / \mathrm{GaAs}$ interfaces, spin-resolved band structures were calculated with results presented in Figs. S3 and S4, respectively. It is well known that under bcc crystal field the $3 d$ states of both Co and Ni split into $e_{g}$ and $t_{2 g}$ orbitals. The two higher energy $e_{g}$ orbitals are not completely filled and are primarily responsible for the net spin polarization and magnetism in these systems. For the purpose of comparison, the band structures of $1 \mathrm{ML}$ non-hybridized Co and Ni taken from the topmost stabilizing layers are included in each figure (see the left columns of Figs. S3 and S4, respectively). The $3 d\left(e_{g}\right)$ orbital occupancies of the stabilizing Co and Ni concentrate within a few flat bands, while that of the interfacial $\mathrm{Co}$ and $\mathrm{Ni}$ are strongly delocalized due to the presence of GaAs. The hybridization with GaAs occur around the $X$ point $(0, \pi, 0)$ of the Brillouin zone. Notably, the hybridization between Co $d$ and GaAs $s p^{3}$ mainly occurs at $\sim 0.6 \mathrm{eV}$ below $E_{\mathrm{F}}$, while that between Ni $d$ and GaAs $s p^{3}$ occurs at $E_{\mathrm{F}}$. This explains why the interface has a much stronger impact on the magnetization of $\mathrm{Ni}$, compared to that of $\mathrm{Co}$ on $\mathrm{GaAs}$. 

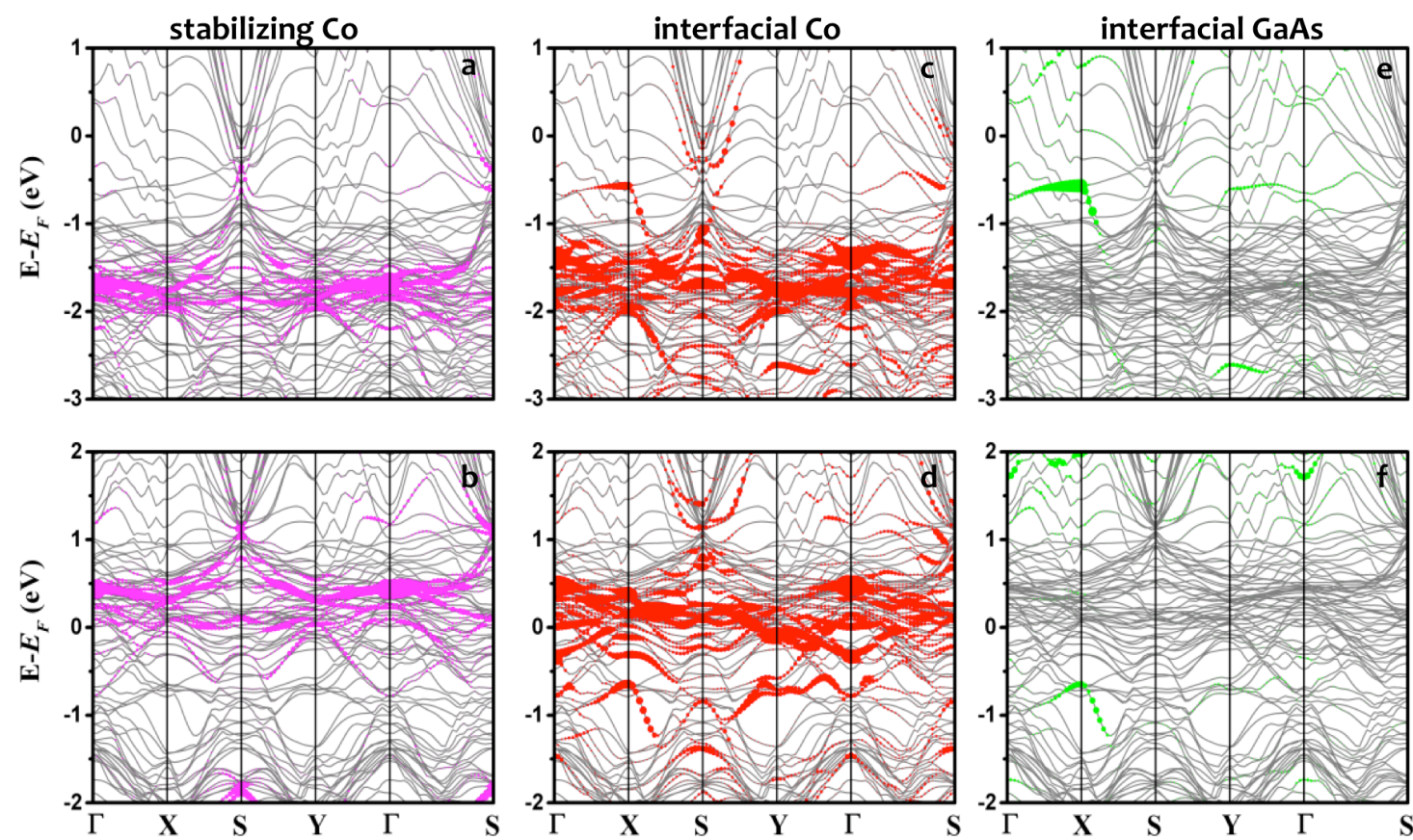

Figure S3. Spin-resolved band structure of (a)-(b) stabilizing Co, (c)-(d) interfacial Co, and (e)-(f) interfacial GaAs of Co/GaAs. Upper and lower rows present the spin-up and spin-down band structure, respectively. The $3 d\left(e_{g}\right)$ of the stabilizing Co and the interfacial Co, and the $4 s$ and $4 p$ orbitals of GaAs are colored pink, red, and green, respectively.


Figure S4. Spin-resolved band structure of (a)-(b) stabilizing Ni, (c)-(d) interfacial Ni, and (e)-(f) interfacial $\mathrm{GaAs}$ of the $\mathrm{Ni} / \mathrm{GaAs}$. Upper and lower rows present the spin-up and spin-down band structure, respectively. The $3 d\left(e_{g}\right)$ of the stabilizing $\mathrm{Ni}$ and the interfacial $\mathrm{Ni}$ and the $4 s$ and $4 p$ orbitals of GaAs are colored pink, red, and green, respectively. 


\section{Charge transfer}

Figure S5 presents the two-dimensional differential electronic density map in the [011] direction of the four superstructures, (a) $7 \mathrm{ML} \mathrm{Fe} / 1 \mathrm{ML} \mathrm{Co}$, (b) $8 \mathrm{ML} \mathrm{Co}$, (c) $7 \mathrm{ML} \mathrm{Co} / 1 \mathrm{ML} \mathrm{Ni}$, and (d) $8 \mathrm{ML} \mathrm{Ni}$ atop GaAs(100). The most prominent charge-transfer at the FM/SC occurs between the terminated $\mathrm{Ga}$ and the interfacial $\mathrm{Co}$ or $\mathrm{Ni}$ atoms and the electron density accumulated at the former is only half of that at the latter. This accumulation quickly diminishes when entering the second FM layer from the substrate, which explains why the hybridization only has a limited effect on the topmost $\mathrm{FM}_{1}$ (see Table I in the main paper).
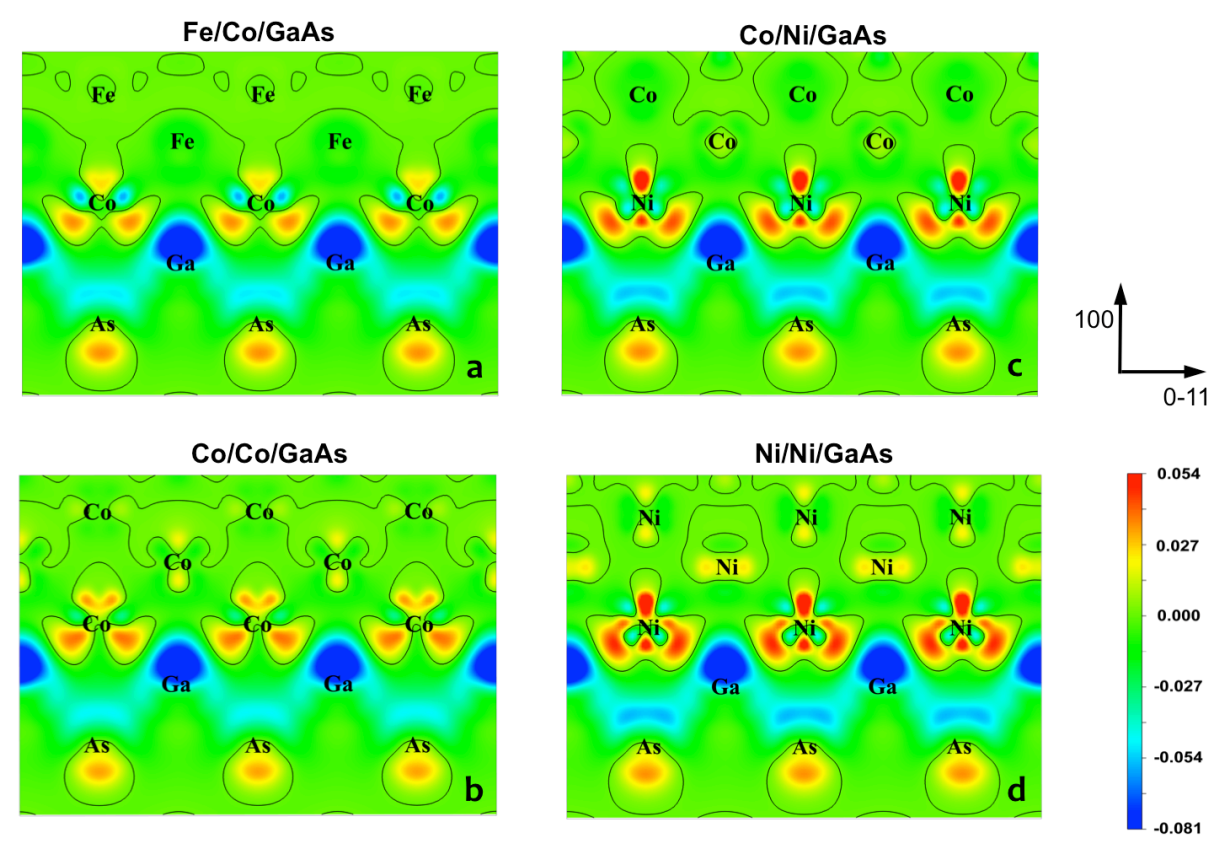

Figure S5. Two-dimensional differential electronic density map in the [011] direction of the four superstructures, (a) $7 \mathrm{ML} \mathrm{Fe} / 1 \mathrm{ML}$ Co, (b) $8 \mathrm{MLCo}$, (c) $7 \mathrm{ML} \mathrm{Co} / 1 \mathrm{ML} \mathrm{Ni}$, and (d) $8 \mathrm{ML} \mathrm{Ni}$ atop GaAs(100). The unit of the scale is $e / \AA^{3}$. 